\title{
Ventriculo-ureteral shunt insertion using percutaneous nephrostomy: a novel minimally invasive option in a patient with chronic hydrocephalus complicated by multiple distal ventriculoperitoneal shunt failures
}

\author{
Ashok Pillai, MD, DNB(NS), ${ }^{1}$ Georgie Mathew, MS, $M C h,{ }^{2}$ Sivasankaran Nachimuthu, MS, $M C h,{ }^{2}$ \\ and Sanjeevan Vasudevan Kalavampara, MS, $\mathrm{MCh}^{2}$
}

Departments of ${ }^{1}$ Neurosurgery and ${ }^{2}$ Urology, Amrita Institute of Medical Sciences, Amrita Vishwa Vidyapeetham University, Kochi, India

\begin{abstract}
The management of ventriculoperitoneal (VP) shunt failure is a common problem in neurosurgical practice. On occasion, extraperitoneal sites for CSF diversion are required when shunting to the peritoneal cavity has failed after multiple attempts. The authors report a novel minimally invasive procedure allowing cannulation of the ureter for the purpose of ventriculo-ureteral (VU) shunting. Sixteen years prior to presentation, this 46-year-old woman had contracted tuberculous meningitis and had chronic hydrocephalus, with multiple distal shunt failures in recent months. A percutaneous nephrostomy was used to pass the distal catheter based on intraoperative retrograde pyelography. Following successful placement of the VU shunt, the patient's hydrocephalus stabilized and she returned to her regular functional status. The only long-term complication noted within 36 months of follow-up was a transient episode of electrolyte disturbance and dehydration associated with a diarrheal illness that responded to adequate hydration and salt supplementation. By its minimally invasive nature, this approach offers a reasonable extraperitoneal alternative after multiple distal shunt catheter failures have occurred.
\end{abstract}

https://thejns.org/doi/abs/10.3171/2016.8.JNS16342

KEY WORDS ventriculo-ureteral shunt; hydrocephalus; VP shunt malfunction; ventriculoperitoneal

$\mathrm{V}$ ENTRICULOPERITONEAL CSF shunting remains the most commonly offered treatment for hydrocephalus, with shunt failure and infection as its main complications. Seventy-seven percent of shunt obstructions involve the ventricular end of the catheter and 22\% the peritoneal end.5,18 Apart from mechanical causes of dysfunction, the absorptive capacity of the peritoneum is another essential element for successful ventriculoperitoneal (VP) shunt function. Peritoneal causes of shunt failure have been described involving either ascites or an abdominal pseudocyst ${ }^{3,10}$ with separate pathophysiology and management options. In both children and adults undergoing chronic shunt treatment for hydrocephalus, prolonged avoidance of the peritoneal cavity is occasionally necessary. Ommaya reservoir tapping, shunt exteriorization, and ventriculosubgaleal shunts in children are often useful in temporary crisis management until the catheter can be returned to the peritoneum. Yet these methods may increase the number of procedures, infection risks, and hospital stay.

Currently, alternative CSF diversion sites include the right atrium, ${ }^{3,10,12}$ pleural space, ${ }^{15}$ gall bladder,${ }^{1,11}$ and ureter. ${ }^{2,4,6,9,13,14,16}$ However, each of these is known to be technically difficult with either high failure rates or their own long-term complications. The ureter has the added advantage of avoiding absorption issues, but theoretically raises concerns of infection ascending from the lower urinary tract, chronic encrustation, potential retrograde reflux of urine into the CSF spaces, and potential long-term fluidelectrolyte imbalance due to chronic loss of CSF. Finally, practical considerations in catheter positioning into the distal ureter and surgical morbidity with previously reported techniques have historically made the ventriculoureteral (VU) shunt less attractive to neurosurgeons. We present our favorable experience using a novel technique for minimally invasive catheter placement into the ureter

ABBREVIATIONS VA $=$ ventriculo-atrial; $V P=$ ventriculoperitoneal; $V U=$ ventriculo-ureteral.

SUBMITTED February 22, 2016. ACCEPTED August 5, 2016.

INCLUDE WHEN CITING Published online November 11, 2016; DOI: 10.3171/2016.8.JNS16342. 
as an attractive option for failed VP shunting and review the existing VU shunt literature.

\section{Case Report}

This 46-year-old woman had a history of tubercular meningitis 16 years earlier that was complicated by hydrocephalus. Her initial right VP shunt functioned adequately with only one revision performed 5 years after placement. Recently, an endoscopic third ventriculostomy was attempted at another institution because of shunt failure, and a left occipital VP shunt was inserted 2 weeks later due to failure of the endoscopic third ventriculostomy. The patient presented to our institution for the first time with worsening level of consciousness, gait insufficiency, and upgaze ocular palsy due to acute hydrocephalus (Fig. 1 left). On exploration, the distal limb of the existing shunt was draining adequately; hence, the peritoneal catheter was repositioned through a fresh laparotomy. The patient's hydrocephalus stabilized within hours, and she was discharged within days.

Four weeks later, the patient presented with acute hydrocephalus. The shunt was exteriorized from the abdominal wound, and the patient's sensorium rapidly improved. The patient then underwent conversion to a ventriculoatrial (VA) shunt tunneled from the left occipital ventricular catheter to the right external jugular vein with a medium-pressure valve. She again became symptomatic after 5 days, and a right frontal Ommaya reservoir was placed for temporary stabilization. The VA shunt was revised using the common facial vein and passage of the distal catheter via the internal jugular vein. Within 48 hours her sensorium again deteriorated, and intermittent Ommaya reservoir aspiration was required. Within 3 days, the shunt was converted to a ventriculopleural shunt through the right second intercostal space, and she had rapid resolution of delirium, upgaze palsy, and urinary incontinence. She was ambulatory at discharge on the 4th day. An outpatient chest radiograph showed right pleural effusion on the 10th day (Fig. 1 right).

Twenty days after ventriculopleural shunt placement, the patient presented again to the emergency department with acute hydrocephalus and symptomatic worsening of the pleural effusion. She was admitted for Ommaya taps and consideration of ventriculo-gall bladder shunting. Ultrasonography of the abdomen revealed a $13-\mathrm{mm}$ gall bladder calculus and poor postprandial emptying of the gall bladder; hence, a ventriculo-gall bladder shunt appeared to be relatively contraindicated. A repeat laparotomy was performed for repositioning of a fresh distal shunt catheter into the subhepatic space. The patient's condition failed to improve, and Ommaya tapping followed by shunt exteriorization was required. Serial CSF cultures remained sterile.

It was decided to proceed with a VU shunt. A preoperative contrast-enhanced CT scan of the abdomen showed no evidence of abdominal or genito-urinary tuberculosis and normal pelvicalyceal anatomy. The urine culture was sterile, and serum urea/creatinine levels were within normal limits. Cystoscopy immediately prior to VU shunting revealed normal mucosa and adequate bladder capacity. A

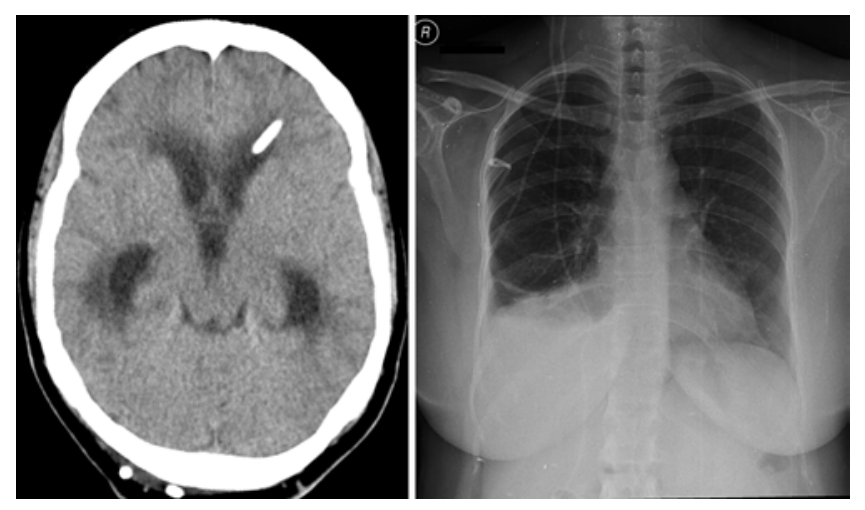

FIG. 1. Left: CT scan at presentation showing ventriculomegaly and adequate position of the left occipital ventricular catheter with an old nonfunctioning catheter in the contralateral ventricle. Right: Chest radiograph obtained 10 days after conversion to a ventriculopleural shunt, showing a right-sided pleural effusion.

retrograde pyelogram was done on the left side (ipsilateral to the existing ventricular catheter) that delineated normal pelvicalyceal and ureteral anatomy, and a ureteral catheter was placed retrograde in the renal pelvis (Fig. 2A). The patient was then turned prone, and a midposterior calyceal puncture was achieved percutaneously under fluoroscopic guidance after dye injection from below (Fig. 2B). A 0.035 -in guidewire was advanced into the distal ureter, and the access tract was dilated up to $10 \mathrm{Fr}$ with Teflon dilators. The ureteral catheter was removed from below. A 20-cm distal VP shunt catheter was passed over the guidewire and placed in the ureter just above the level of the sacroiliac joint (Fig. 2C and D). The preexisting left occipital ventricular catheter was connected to a fresh distal catheter with a medium-pressure valve (Surgiwear springvalve) that was tunneled subcutaneously through the back to the left flank and connected with a separate connector to the distal ureteral catheter after confirming adequate CSF drainage.

The patient's sensorium improved gradually over the next week without further Ommaya tapping. She became ambulatory within 7 days and was discharged on the 9th postoperative day, obeying commands with resolved upgaze palsy. At 3 months, her gait and memory were normal, and she was able to perform all of her regular activities without disability. A follow-up intravenous urogram obtained at 20 days showed good renal function and a well-placed shunt catheter in the distal ureter. The patient has since remained asymptomatic for hydrocephalus, and she has not experienced any urinary colic or infections for 36 months. She developed hyponatremia (serum sodium $124 \mathrm{mEq} / \mathrm{L}$ ) and dehydration following an acute diarrheal illness at 36 months from the VU shunt procedure. The electrolyte imbalance and symptoms resolved within days after intravenous hydration and oral salt supplementation. Although the CT findings at 36 months showed features of mild shunt overdrainage (Fig. 3), the patient was not symptomatic for this.

\section{Discussion}

Initial attempts to divert CSF to the urinary tract be- 

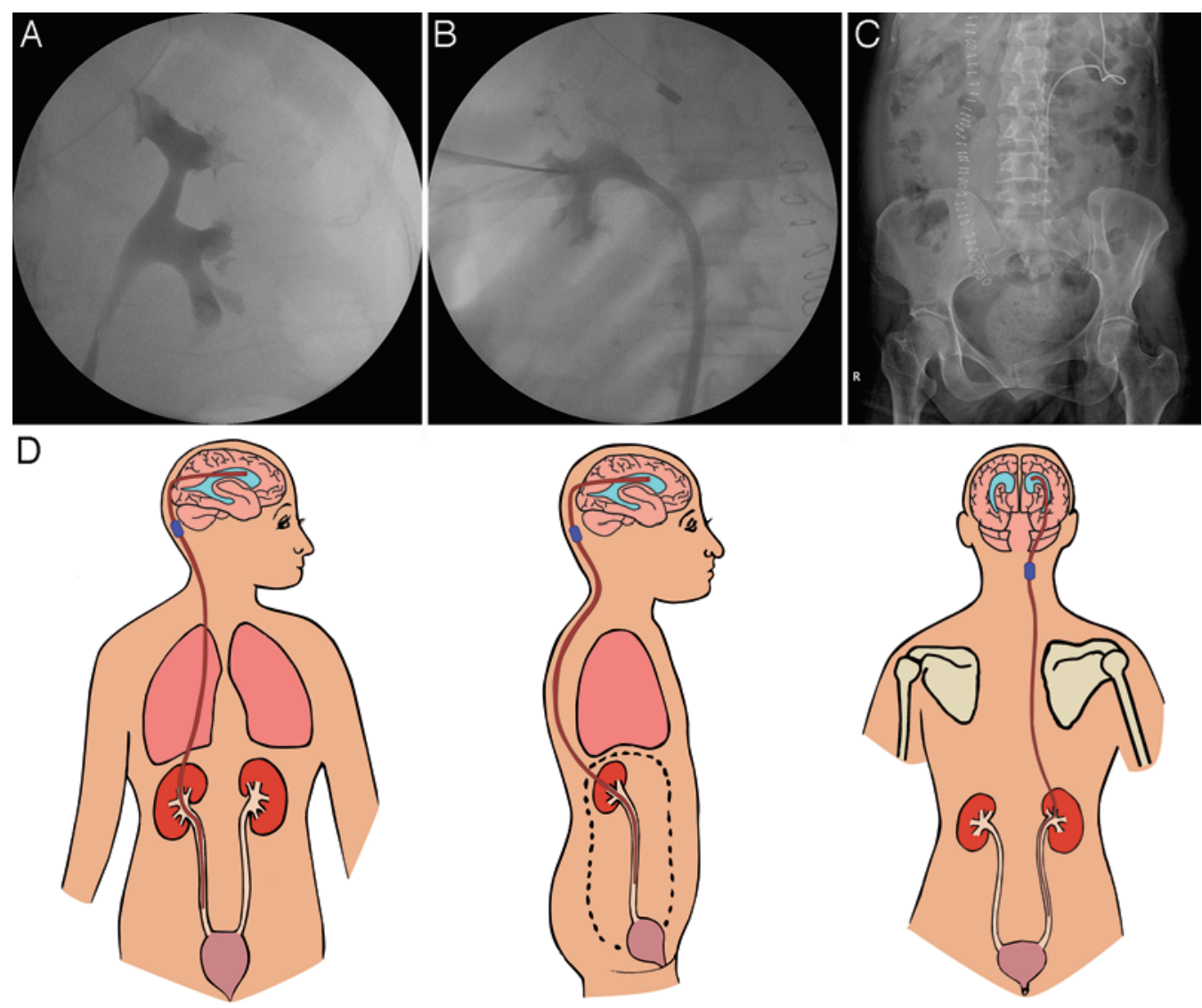

FIG. 2. A: Retrograde pyelogram of the left kidney in the supine position to define the pelvicalyceal anatomy intraoperatively and choose the calyx for entry. B: Middle calyceal puncture and guidewire insertion in the prone position. C: Final shunt catheter position via the middle calyx, reaching the mid ureter. Skin staples seen are from the previous midline laparotomy for peritoneal catheter repositioning 10 days prior. D: Schematic diagram of percutaneous nephrostomy access for VU shunt placement. Copyright Amrita Institute of Medical Sciences (Fig. 2D). Published with permission. Figure is available in color online only.

gan well before the establishment of VP shunting. In 1925, Heile first reported a series of 4 patients who underwent ureterodural anastomosis following unilateral nephrectomy. ${ }^{9}$ One patient died intraoperatively, and the remaining 3 patients experienced at least temporary improvement. Subsequent attempts at ureterodural anastomosis were associated with high mortality rates. ${ }^{4,6}$ Attempts during the subsequent decades included uretero-arachnoid anastomosis ${ }^{13,17}$ but were again met with high mortality. Torkildsen detailed his technique of ventriculocisternostomy for noncommunicating hydrocephalus and dismissed the uretero-arachnoid anastomosis, stating, "This ingenious operation is connected with a very high mortality and has never gained considerable practical importance because of its operative and post-operative complications." 21

Finally, it was Matson who first reported a successful, rapid recovery from postmeningitic communicating hydrocephalus in an 8-year-old child following lumboureteral shunting in $1949 .{ }^{14}$ In this novel technique, a unilateral nephrectomy was performed, followed by insertion of a polyethylene shunt tube between the lumbar CSF space and the ureter. Notably, there were no electrolyte disturbances or urinary dysfunction noted up to 6 months following the operation, and the child made a remarkable recovery. The author went on to successfully perform the same urinary CSF diversion procedure in 3 hydrocephalic infants (3-5 months of age) and noted, "Preliminary observations indicate that in infants $2-3$ grams of salt daily should be added to the diet to prevent excessive chloride loss." ${ }^{14}$ By the 1960s, the VA shunt had become more standardized and urinary CSF diversion remained as a backup procedure in more complicated hydrocephalus.

The next phase in the development of VU shunts focused on avoiding nephrectomy. In 1980, Smith et al. described a technique involving low ureteral transection for VU shunt catheter placement combined with primary reimplantation of the ureter. ${ }^{20}$ This procedure avoided the need for nephrectomy and probably facilitated diversion of the urine stream away from the shunt. Pittman et al. described a similar technique in 1992 in which the ureter was transected $4 \mathrm{~cm}$ from the ureteral insertion in the bladder, and the distal ureter was cannulated with the abdominal end of the VP shunt, fixed to the psoas muscle with direct reimplantation of the proximal ureter segment into the bladder. When performed in an 8-year-old child, they noted no electrolyte disturbances. ${ }^{16}$

Biddle measured urinary protein and sodium output using a valved VA shunt tube and the Matson technique. ${ }^{2}$ He concluded that a valved VU shunt might result in less electrolyte and protein imbalance, though this conclusion 


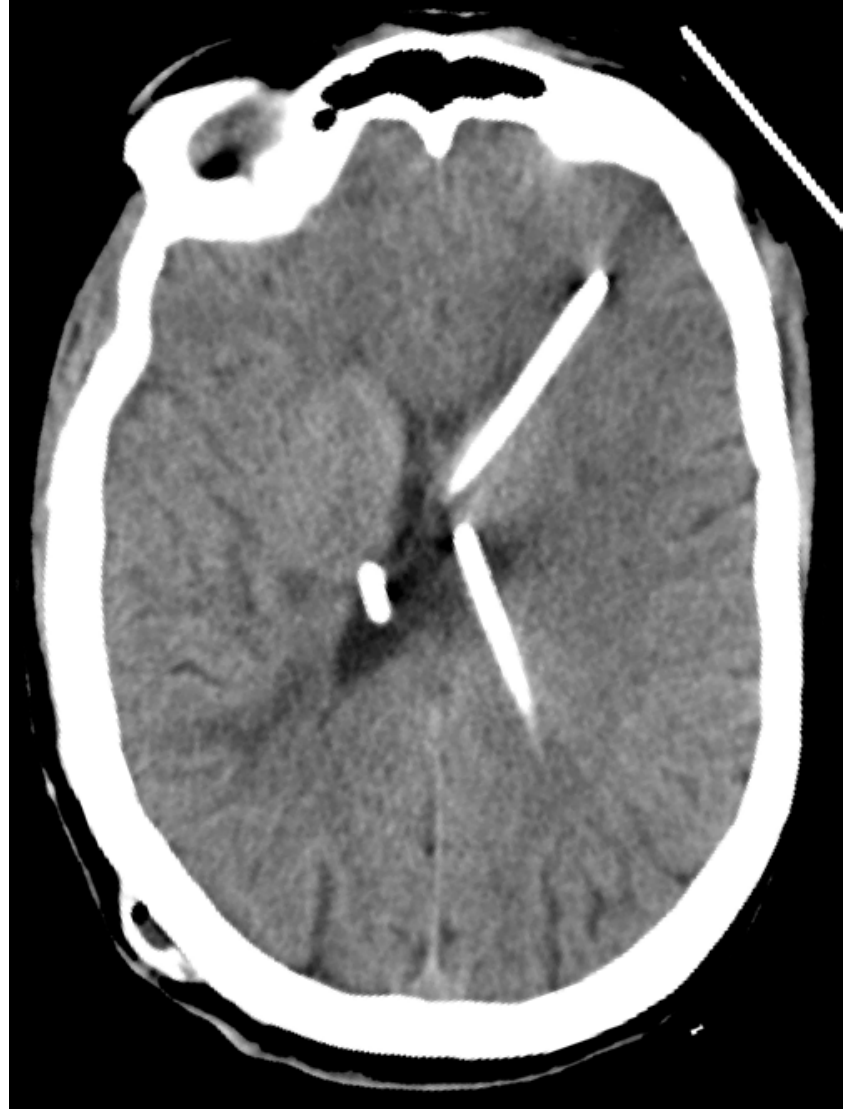

FIG. 3. CT scan obtained 3 years after VU shunt placement. The left lateral ventricular catheter connects to the $\mathrm{VU}$ shunt. The right-sided posterior catheter is from a previous VP shunt and is not connected distally; the right-sided anterior catheter is from the retained Ommaya reservoir.

was based on a single patient's data. Nevertheless, the hazards of severe fluid and electrolyte depletion in the event of vomiting, diarrhea, or otherwise restricted fluid and salt intake were emphasized. Approximately $400-500 \mathrm{ml}$ of CSF is produced daily in adults and averages $6 \mathrm{ml} / \mathrm{hr}$ in children. This is roughly equivalent to the average daily physiological insensible fluid loss through the respiratory tract and skin, which is normally accommodated for in the natural homeostatic mechanisms of the hypothalamus. Therefore normal thirst mechanisms probably adjust for the additional daily fluid loss. This volume of CSF would equate to an additional excretion of $3.6 \mathrm{~g}$ of sodium chloride, compared with the daily 11-13 g physiological urinary sodium excretion. The electrolyte imbalance noted in our patient following an acute diarrheal illness is previously well described, and patients should be counseled to maintain increased hydration and oral salt supplementation in such situations.

Potential advantages of VU shunting include that 1) it is not dependent on CSF absorption and 2) it can remain patent for a long time by avoiding the issues related to omental or peritoneal reaction. Our new percutaneous technique is easy to perform with a short operative time and with minimal invasiveness. Of note, in subsequent cases (unpublished data), we have found that entry through the superior calyx allows easier passage of the catheter into the ureter. Distal VU shunt revision, if ever required, should theoretically be easy to perform through the same access. Furthermore, shunt function could potentially be evaluated with injection of dye (e.g., methylene blue, fluorescein) into the shunt reservoir and detection in the urine. All previous techniques for catheter placement into the distal ureter involved division and reimplantation of the proximal ureter into the bladder. This is a cumbersome procedure requiring longer operative times, lower abdominal incision, and extensive periureteral and perivesical dissection.

Potential long-term concerns with our technique include shunt encrustation, ${ }^{8}$ biofilm formation on the shunt, ${ }^{22}$ and erosion of the ureteral wall due to the long-term presence of a foreign body. ${ }^{19}$ Periodic evaluation with imaging either with abdominal radiography or noncontrast CT may be advisable. However, the inert nature of currently available CSF shunt catheters should mitigate this problem. Also of concern is the potential reflux of urine and/or ascending infection into the ventricles. However, we feel that the use of a one-way valve, intervening connectors, significant catheter length, and a siphoning effect should all help avoid this problem. During 36 months of follow-up, our patient certainly did not exhibit any symptoms indicative of such a reflux, although this would need to be confirmed in a larger series. It is important to rule out high intravesical pressure due to neurogenic bladder, which may coexist in hydrocephalic patients. Therefore, the procedure is probably contraindicated if the preoperative radiological evaluation reveals hydroureteronephrosis, which was ruled out in our patient based on preoperative abdominal CT scanning findings. In such patients, further urodynamic evaluation is warranted for therapeutic interventions that prevent further renal deterioration. Finally, this procedure is probably contraindicated in patients with active urinary tract infection or a history of recurrent infections.

Since the late 1960 s, the peritoneal cavity has remained the preferred site for CSF shunt insertion. Other sites include the right atrium, ${ }^{12}$ pleural space, ${ }^{15}$ gall bladder,, 11 and even the fallopian tubes. ${ }^{11}$ Yet these sites are all associated with higher failure rates or their own attendant complications. Therefore, every effort should be made to salvage the peritoneal cavity for the distal shunt catheter insertion. Once distal shunt malfunction has been demonstrated, the various mechanical factors like shunt catheter fracture or disconnection should first be ruled out. Subsequently the peritoneal cause for failure should be classified as either pseudocyst formation or ascites due to malabsorption. ${ }^{10}$ Laparoscopic or open shunt catheter repositioning is efficacious in the former while extraperitoneal shunt placement is indicated in the latter. ${ }^{10}$

\section{Conclusions}

We have reported this novel minimally invasive technique as a viable option in patients with hydrocephalus complicated by a peritoneal cavity that, after multiple attempts, has proven to be inadequate for CSF shunting. The percutaneous technique avoids nephrectomy and significantly reduces the morbidity, which in our initial experience makes this the next most convenient site for CSF di- 
version after the peritoneum. Studies with larger numbers of patients with long-term follow-up will be required to adequately assess the long-term risk-benefit ratio. Yet, it is entirely possible that the risks may be insignificant, as all reports of these shunt-related complications are described in the era of nephrectomy-associated VU shunting. This procedure can therefore be considered as a viable step into the algorithm of distal shunt revision in shunt-dependent hydrocephalus.

\section{Acknowledgments}

We wish to acknowledge Dr. Murugankutty Gopalan of the anatomy department/medical illustrations, AIMS, Kochi, for his schematic diagram (Fig. 2D).

\section{References}

1. Aldana PR, James HE, Postlethwait RA: Ventriculogallbladder shunts in pediatric patients. J Neurosurg Pediatr 1:284-287, 2008

2. Biddle A: Lumbar arachnoid-ureterostomy combining the Matson technique and the Pudenz-Heyer valve. Report of a case. J Neurosurg 24:760-761, 1966

3. Chidambaram B, Balasubramaniam V: CSF ascites: a rare complication of ventriculoperitoneal shunt surgery. Neurol India 48:378-380, 2000

4. Christopher F: Ureterodural anastomosis. Surg Clin North Am 9:473-479, 1929

5. Cozzens JW, Chandler JP: Increased risk of distal ventriculoperitoneal shunt obstruction associated with slit valves or distal slits in the peritoneal catheter. J Neurosurg 87:682686, 1997

6. Davidoff LM, Bancroft FW: Ureterodural anastomosis for the treatment of hydrocephalus: Report of a case. Arch Surg 25:550-554, 1932

7. Drake JM, Sainte-Rose C, DaSilva M, Hirsch JF: Cerebrospinal fluid flow dynamics in children with external ventricular drains. Neurosurgery 28:242-250, 1991

8. el-Faqih SR, Shamsuddin AB, Chakrabarti A, Atassi R, Kardar AH, Osman MK, et al: Polyurethane internal ureteral stents in treatment of stone patients: morbidity related to indwelling times. J Urol 146:1487-1491, 1991

9. Heile B: Über neue operative Wege zur Druckentlastung bei angeborenem Hydrocephalus (Ureter-Duraanastomose). Zbl Chir 52:2229-2236, 1925

10. Karyattil R, Steinbok P, Singhal A, Cochrane DD: Ascites and abdominal pseudocysts following ventriculoperitoneal shunt surgery: variations of the same theme. J Neurosurg (5 Suppl Pediatrics) 106:350-353, 2007
11. Ketoff JA, Klein RL, Maukkassa KF: Ventricular cholecystic shunts in children. J Pediatr Surg 32:181-183, 1997

12. Keucher TR, Mealey J Jr: Long-term results after ventriculoatrial and ventriculoperitoneal shunting for infantile hydrocephalus. J Neurosurg 50:179-186, 1979

13. Lehman EP: Uretero-arachnoid (ureterodural) anastomosis. With report of three cases. Ann Surg 100:887-905, 1934

14. Matson DD: A new operation for the treatment of communicating hydrocephalus; report of a case secondary to generalized meningitis. J Neurosurg 6:238-247, 1949

15. Piatt JH Jr: How effective are ventriculopleural shunts? Pediatr Neurosurg 21:66-70, 1994

16. Pittman T, Steinhardt G, Weber T: Ventriculo-ureteral shunt without nephrectomy. Br J Neurosurg 6:261-263, 1992

17. Sachs E: Hydrocephalus. Analysis of ninety-eight cases. J Mt Sinai Hosp 9:767-791, 1942

18. Sainte-Rose C: Shunt obstruction: a preventable complication? Pediatr Neurosurg 19:156-164, 1993

19. Singh I, Gupta NP, Hemal AK, Aron M, Seth A, Dogra PN: Severely encrusted polyurethane ureteral stents: management and analysis of potential risk factors. Urology 58:526-531, 2001

20. Smith JA Jr, Lee RE, Middleton RG: Ventriculoureteral shunt for hydrocephalus without nephrectomy. J Urol 123:224-226, 1980

21. Torkildsen A: A follow-up study 14 to 20 years after ventriculocisternostomy. Acta Psychiatr Scand 35:113-121, 1960

22. Tunney MM, Keane PF, Jones DS, Gorman SP: Comparative assessment of ureteral stent biomaterial encrustation. Biomaterials 17:1541-1546, 1996

\section{Disclosures}

The authors report no conflict of interest concerning the materials or methods used in this study or the findings specified in this paper.

\section{Author Contributions}

Conception and design: Pillai, Mathew, Kalavampara. Acquisition of data: Mathew, Nachimuthu. Drafting the article: Pillai, Mathew, Nachimuthu. Critically revising the article: Pillai, Mathew, Kalavampara. Reviewed submitted version of manuscript: Pillai, Mathew, Nachimuthu. Approved the final version of the manuscript on behalf of all authors: Pillai. Operating surgeons: Pillai, Mathew, Kalavampara.

\section{Correspondence}

Ashok Pillai, Department of Neurosurgery, Amrita Institute of Medical Sciences, AIMS Ponekkara PO, Kochi, Kerala 682 041, India. email: brashok@aims.amrita.edu. 